\title{
Analysis of pelvic floor electrical physiological parameters in nulliparous women with stress urinary incontinence
}

\author{
Xiaoxia Chang, Huan Ge, Guihua Ye, Xiaojie Quan, Wei Shen, Chunzi Zhang, Mengyao Huan, Jie Wu \\ The State Key Laboratory of Reproductive Medicine, Department of Obstetrics and Gynecology, Women's Rehabilitation Medical Center, Jiangsu \\ Province People's Hospital, the First Affiliated Hospital of Nanjing Medical University, Nanjing 210036, China \\ Contributions: (I) Conception and design: J Wu; (II) Administrative support: X Chang, H Ge; (III) Provision of study materials or patients: H Ge, \\ X Chang, X Quan; (IV) Collection and assembly of data: G Ye, W Shen, C Zhang, M Huan; (V) Data analysis and interpretation: X Chang; (VI) \\ Manuscript writing: All authors; (VII) Final approval of manuscript: All authors. \\ Correspondence to: Jie Wu, MD, PhD. The State Key Laboratory of Reproductive Medicine, Department of Obstetrics and Gynecology, Women's \\ Rehabilitation Medical Center, Women's Rehabilitation Medical Center, Jiangsu Province People's Hospital, the First Affiliated Hospital of Nanjing \\ Medical University, Jiangdong Road 368, Nanjing 210036, China. Email: wujeidoc60@163.com.
}

Background: To investigate the changes in pelvic floor electrical physiological parameters in nulliparous women with stress urinary incontinence (SUI).

Methods: A retrospective survey was conducted on 922 women aged 20-40 years who received health examinations at the First Affiliated Hospital of Nanjing Medical University between July 2017 and December 2019. The women were split into two groups: those who had SUI ( $n=87)$ and those that did not $(n=835)$. Questionnaires and pelvic floor electrical physiological indexes were collected.

Results: The mean age of the group of women with SUI was 29.77 years, while the mean age of the group of women with no SUI was 24.49 years. The body mass indexes (BMI) of the women with SUI were significantly higher than those of the women with no SUI. Importantly, the normal rates of type I and II fibers in the women with SUI were obviously lower than those in the women with no SUI. Moreover, the vaginal dynamic pressure in the women with SUI was significantly lower than in the women with no SUI. The study also found that the incidence of SUI in nulliparous women was higher in those aged 30-40 and that both low-weight and obese women had an increased risk of SUI. Type I and type II muscle fibers were more abnormal in the women with SUI than in those with no SUI. In multivariate logistic regression, the ages, BMIs, and type I fiber indexes of nulliparous women were related to SUI.

Conclusions: Nulliparous women have a higher rate of SUI. Compared to women with no SUI, the possible potential risk factors are age ( $>30$ years), higher BMI, and abnormal type I muscle fiber of the pelvic floor.

Keywords: Stress urinary incontinence; pelvic floor electrical physiological parameters; nulliparous women; clinical study

Submitted Sep 04, 2020. Accepted for publication Feb 10, 2021.

doi: $10.21037 /$ tau-20-1235

View this article at: http://dx.doi.org/10.21037/tau-20-1235

\section{Introduction}

Urinary incontinence (UI) is a problem affecting adult women worldwide. Approximately $30.9 \%$ of women aged 20 or older in China have experienced UI, with stress urinary incontinence (SUI) being the most common type (1).
The U.S. reported that the prevalence of UI among women aged $30-90$ was $45 \%$ (2), while the prevalence of UI among women aged $\geq 18$ years in four European countries was reported as $35 \%$. These data suggest that the condition seriously affects women's quality of life (3). SUI can cause psychological and social problems, such as depression, social 
loneliness, reduced self-confidence, family disharmony, and other related health problems, and it has become one of the most important chronic diseases affecting public health (4).

Although pregnancy and childbirth history are considered independent risk factors for UI, other risk factors include post-menopausal status, obesity, and pelvic surgery. Most studies on UI have been mainly based on postpartum women, but SUI also occurs in non-fertile young women.

Pelvic floor surface electromyography is a non-invasive technique that collects muscle motor potentials through skin surface electrodes to observe muscle recruitment patterns (5). It can detect abnormalities in the pelvic floor of women, thereby enabling early detection and prevention of pelvic floor disease. Some studies have reported that the surface electromyographic parameters of the pelvic floor muscles change before the onset of pelvic floor dysfunction (PFD), which occurs before the onset of clinical symptoms. The main objective of the present study is to investigate the changes in the pelvic floor electrical physiological parameters of nulliparous women with SUI. We present the following article in accordance with the STROBE reporting checklist (available at http://dx.doi.org/10.21037/tau-201235).

\section{Methods}

\section{Participants}

A retrospective survey, via interviews, was conducted on 922 nulliparous women who received health examinations at the First Affiliated Hospital of Nanjing Medical University between July 2017 and December 2019. Questions regarding stress ("Do you leak urine with activities such as coughing, sneezing, or running?") was used to determine participant eligibility. Also, symptoms of urge incontinence ("Does urine leak when you rush/hurry to the toilet?") should be excluded. Only participants predominantly suffering from SUI were recruited. The women were divided into two groups: those with SUI $(n=87)$ and those without $(\mathrm{n}=835)$. All patients provided standardized information (age, height, weight, etc.) and gave informed consent. Pelvic floor electrical physiological parameters were collected and analyzed.

The inclusion criteria were $20-40$ years old and tolerance of gynecological examination. Patients with a history of one or more births, pelvic organ prolapse (POP), known or suspected pregnancy, unexplained irregular vaginal bleeding, cognitive impairment rendering pelvic floor function assessment impossible, urogynecological and gynecological surgeries history or other types of UI were excluded.

This study was conducted in accordance with the Declaration of Helsinki (as revised in 2013). This study was conducted with approval from the Ethics Committee of Women's Rehabilitation Medical Center (2014-SR146). Written informed consent was obtained from all participants.

\section{Pelvic floor electrical physiological parameters}

Phenix U4, a neuromuscular stimulation therapy instrument, was used by trained obstetrics and gynecology medical personnel to detect pelvic floor electrophysiological indicators. The final assessment of the pelvic floor electrophysiological indicators was undertaken by professional pelvic floor gynecology physicians.

\section{Observation parameters}

Pelvic floor electrical physiological parameters, including pelvic floor muscles, type I vaginal dynamic pressure, and type II muscle fiber strength and fatigue.

\section{Pelvic floor type I and type II muscle fiber strength testing methods and evaluation standards}

Type I muscle fiber contraction was continued until more than $40 \%$ of its maximum intensity had been reached, continues to a level $0,0 \mathrm{~S}$ last $1 \mathrm{~S}$ for level 1 , continues to level 2, $2 \mathrm{~S}$ for $3 \mathrm{~S}$ to level 3, continues to level 4, $4 \mathrm{~S}$ for $5 \mathrm{~S}$ and above level 5 . The number of times that the contraction strength of the muscle fibers was greater than $60 \%$ of the maximum value, and the duration of 1 time was 1 , the duration of 2 times was 2 , the duration of 3 times was 3 , the duration of 4 times was 4 , and the duration of 5 times or more was 5 . The judgment criteria for muscle force abnormalities assessed grade $4-5$ as normal and grade $0-3$ as abnormal. The measurement of muscle fatigue was the percentage decrease between the peak of the contraction curve and the peak of the end point at $5 \mathrm{~S}(6)$.

\section{Measurement of vaginal dynamic pressure}

Vaginal dynamic pressure was measured by placing a balloon pressure probe into the vagina. The dynamic pressure of the maximum contraction of the vagina was then measured by the pressure converter; $80-150 \mathrm{cmH}_{2} \mathrm{O}\left(1 \mathrm{cmH}_{2} \mathrm{O}=\right.$ 
Table 1 Clinical characteristics and pelvic floor electrical physiological parameters

\begin{tabular}{|c|c|c|c|}
\hline Variables & No-SUI $(n=835)$ & SUI $(n=87)$ & $P$ \\
\hline $\mathrm{BMI}\left(\mathrm{kg} / \mathrm{m}^{2}\right)$ & $21.56 \pm 3.04$ & $22.83 \pm 4.50$ & 0.012 \\
\hline Gravidity & $0.42 \pm 0.71$ & $0.44 \pm 0.69$ & 0.824 \\
\hline Type I fiber, n (\%) & & & 0.002 \\
\hline Abnormal & $462(53.33)$ & $63(72.41)$ & \\
\hline Type II fiber, n (\%) & & & 0.023 \\
\hline Normal & $452(54.13)$ & $36(41.38)$ & \\
\hline Abnormal & $383(45.87)$ & $51(58.62)$ & \\
\hline Abnormal & $534(63.95)$ & $52(59.77)$ & \\
\hline Type II fatigue, n (\%) & & & 0.479 \\
\hline Normal & $644(77.13)$ & $70(80.46)$ & \\
\hline Abnormal & $191(22.87)$ & $17(19.54)$ & \\
\hline Vaginal dynamic pressure $(\mathrm{mmHg})$ & $83.88 \pm 19.18$ & $77.87 \pm 17.52$ & 0.005 \\
\hline
\end{tabular}

$0.098 \mathrm{~Pa}$ ) was normal, while $<80 \mathrm{cmH}_{2} \mathrm{O}$ was abnormal (6).

\section{Statistical analysis}

All statistical analyses were performed using Stata version 17.0. Data were analyzed using descriptive statistics and multivariate logistic regression. Measurement data conforming to the normal distribution were expressed as the mean and standard deviation, and comparisons were made by $t$-test. Odds ratios (ORs) and $95 \%$ confidence intervals were calculated. All tests were two sided, with $\mathrm{P}<0.05$ considered statistically significant.

\section{Results}

\section{Clinical characteristics and pelvic floor electrical physiological parameters}

A total of 922 participants were recruited in the two groups. The first group included 87 women with SUI and the second group included 835 women with no SUI. The prevalence of SUI was $10.42 \%$. The mean age of the group of women with SUI was 29.77 years, while that of the group of women with no SUI was 24.49 years. Age and BMI were significantly higher in the women with SUI than in the women with no SUI ( $\mathrm{P}=0.000$ and 0.012 ; see Table 1).

The normal rates of type I and type II fibers in the group of women with SUI were obviously lower than those in the group of women with no SUI $(\mathrm{P}=0.002$ and 0.023 ; see Table 1). However, there was no difference between the two groups with respect to type I and II muscle fatigue ( $>>0.05$; see Table 1$)$. Vaginal dynamic pressure in the group of women with SUI was significantly lower than in the group of women with no SUI $(77.87 \pm 17.52$ and 83.88 $\pm 19.18 \mathrm{mmHg} ; \mathrm{P}=0.005)$.

\section{Factors associated with SUI in nulliparous women}

The study explored the risk factors for nulliparous women with SUI in univariate analyses and variables. With increasing age, the incidence of SUI in nulliparous women, especially those aged 30-40, also increased (age 31-35: OR 2.20, 95\% CI: 1.00-4.80, $\mathrm{P}=0.048$; age 36-40: OR 3.79, 95\% CI: $1.46-9.85, \mathrm{P}=0.006)$. However, among those aged 25-30, the incidence of SUI did not increase (OR 1.05, 95\% CI: 0.52-2.16, P=0.879). Being underweight and obese were both associated with an increased risk of SUI (underweight: 
OR 2.15, 95\% CI: 1.01-4.63, P=0.048; overweight: OR 2.19, $95 \%$ CI: $1.25-3.87, \mathrm{P}=0.007$ and OR $3.70,95 \% \mathrm{CI}$ : 1.72-7.91, $\mathrm{P}=0.001)$.

There were greater abnormalities of the type I and type II fibers in the group of women with SUI than in the group with no SUI (type I fiber: OR 2.12, 95\% CI: 1.30-3.46, $\mathrm{P}=0.003$; type II fiber: OR $1.67,95 \% \mathrm{CI}$ : $1.07-2.62, \mathrm{P}=0.024)$. As shown in Table 2, the abnormal rate of vaginal dynamic pressure was significantly lower in the group of women with SUI than in the group with no SUI (OR 1.71, 95\% CI: 1.25-2.31, P=0.001). However, there was no difference between the two groups with respect to muscle fatigue (type I fatigue: OR 0.56, 95\% CI: 0.16-2.01, $\mathrm{P}=0.373$; type II fiber: OR $0.82,95 \%$ CI: $0.47-1.42$, $\mathrm{P}=0.470$ ).

Multivariate logistic regression was used for primary screening, followed by multivariate logistic regression analysis of the potential factors for SUI in nulliparous women. The possible potential risk factors included age above 30 (age 31-35: OR 2.64, 95\% CI: 1.18-5.90, $\mathrm{P}=0.018$; age $36-40$ : OR 4.84, 95\% CI: $1.80-13.01$, $\mathrm{P}=0.002$ ), BMI (overweight: OR 1.90, 95\% CI: 1.33-4.32, $\mathrm{P}=0.004$; obese: $\mathrm{OR} 3.70,95 \% \mathrm{CI}: 1.68-8.14, \mathrm{P}=0.003)$, and type I fibers (OR 1.20, 95\% CI: 1.16-3.70, P=0.013) (see Table 2). However, other pelvic floor electrical physiological parameters without type I fibers had no significant association (see Table 2).

\section{Discussion}

UI, which is one of the main diseases affecting humankind, occurs twice as often in women as in men and is more common as women get older (7). SUI is the most common subtype of UI. Common risk factors for UI are postmenopausal status, pregnancy, and childbirth history (8). Many studies have suggested that SUI is found almost exclusively in elderly women and women who have given birth, but epidemiological studies have reported that younger women and nulliparous women also experience UI episodes (9) and that the risk factors are therefore unknown.

The prevalence of UI subtypes has been reported as ranging between $6.2 \%$ and $42.2 \%$ in young women (10-14). However, previous studies only focused on young sportswomen with SUI $(9,15)$. The results of the present study identified that the prevalence of SUI in 922 nulliparous women was $10.42 \%$. In recent studies, age has been found to be a strong risk factor for SUI. The present study also found that the incidence of SUI increased with age, particularly above the age of 30 in nulliparous women [the same results were reported in Nygaard's study (2)]. However, in the 26-30 age group, the risk of SUI was not significantly increased compared to that of the 20-25 age group (OR 1.05, $\mathrm{P}=0.879$ ). Minassian et al. (16) found that SUI was most prevalent at around 40 years of age, whereas the present study suggests that the risk of SUI between 36 and 40 years of age is highest.

Obesity is a well-known risk factor for SUI and may be associated with increased intra-abdominal pressure. As shown in the present study, women with BMI $\geq 24$ had a higher risk of SUI. However, underweight nulliparous women also had a higher risk of SUI: the odds ratios showed differences with women with normal BMI (OR 2.15, $\mathrm{P}=0.048)$. Therefore, for nulliparous women, maintaining a healthy weight is an effective way of preventing SUI. Most studies have also emphasized that weight loss is the first-line recommendation for all UI subtypes (17).

In recent years, the application of electrophysiological technology has become the focus of the diagnosis of female pelvic floor disease. Pelvic floor surface electromyography can detect abnormalities in the pelvic floor of women, thereby enabling early detection and prevention of pelvic floor disease. However, the current research on UI in non-fertile women is mainly based on epidemiological investigations, and there are few studies on pelvic floor electrical physiological parameters. The present study found that there were greater abnormalities in the type I and type II muscle fibers of women with SUI than in those with no SUI $(\mathrm{P}=0.002$ and 0.023$)$. Nulliparous women with SUI were also found to have lower vaginal dynamic pressure than those with no SUI, which was also found by Shishido et al. (18).

According to the multivariate logistic regression analysis, the possible risk factors for SUI in nulliparous women include age ( $>30$ years old), higher BMI index (overweight: OR 1.90, $\mathrm{P}=0.004$; obese: $\mathrm{OR} 3.70, \mathrm{P}=0.003)$, and type I muscle fibers (OR 1.20). A decrease in type I muscle strength also leads to an increased incidence of SUI in nulliparous women.

This study is a retrospective study and has some limitations. The relationships between occupation, exercise habits, eating habits, chronic diseases, urinary incontinence, and pelvic floor electrical physiological parameters were not addressed. Further, due to time limitations, quality of life was not analyzed. In future research, the research plan will be improved and the sample size expanded to obtain more pelvic floor data for nulliparous women, thereby providing 
Table 2 Factors associated with SUI in nulliparous women

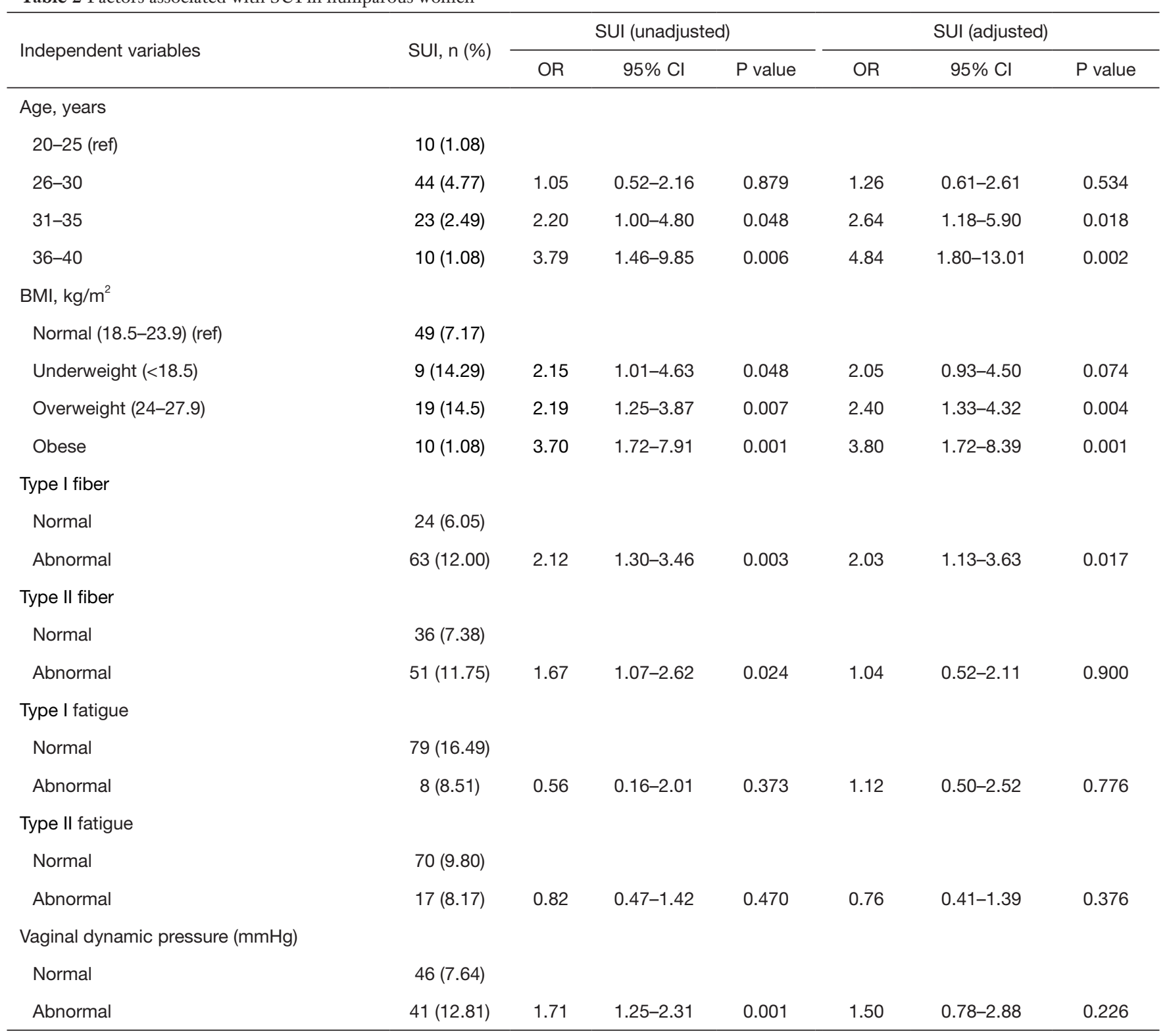

better evidence-based medical recommendations for clinical practice.

\section{Conclusions}

The incidence of SUI in nulliparous women is $10.42 \%$. The risk factors for SUI are age ( $>30$ years old), BMI, and type I muscle fibers. Maintaining a healthy weight and improving type I muscle strength can prevent SUI in nulliparous women.

\section{Acknowledgments}

The authors wish to express their sincere gratitude to all investigators who have actively participated in this study: Department of Obstetrics \& Gynecology, The First Affiliated Hospital of Nanjing Medical University (study design, investigation, and data collection): Danhua $\mathrm{Pu}, \mathrm{Lin}$ li, Yanfang Zhang.

Funding: The study was supported by Jiangsu Innovation Team Project (CXTDA2017004), Maternal and Child 
Health Research Projects in Jiangsu Province (F201722), Jiangsu Women and Children Healthcare Project (FXK201701), Jiangsu Department of Science and Technology (BM2019214), and PAPD of Jiangsu High Education Institutions.

\section{Footnote}

Reporting Checklist: The authors have completed the STROBE reporting checklist. Available at http://dx.doi. org/10.21037/tau-20-1235

Data Sharing Statement: Available at http://dx.doi. org/10.21037/tau-20-1235

Conflicts of Interest: All authors have completed the ICMJE uniform disclosure form (available at http://dx.doi. org/10.21037/tau-20-1235). The authors have no conflicts of interest to declare.

Ethical Statement: The authors are accountable for all aspects of the work in ensuring that questions related to the accuracy or integrity of any part of the work are appropriately investigated and resolved. This study was conducted in accordance with the Declaration of Helsinki (as revised in 2013). This study was conducted with approval from the Ethics Committee of Women's Rehabilitation Medical Center (2014-SR-146). Written informed consent was obtained from all participants.

Open Access Statement: This is an Open Access article distributed in accordance with the Creative Commons Attribution-NonCommercial-NoDerivs 4.0 International License (CC BY-NC-ND 4.0), which permits the noncommercial replication and distribution of the article with the strict proviso that no changes or edits are made and the original work is properly cited (including links to both the formal publication through the relevant DOI and the license). See: https://creativecommons.org/licenses/by-nc-nd/4.0/.

\section{References}

1. Zhu L, Lang J, Liu C, et al. The epidemiological study of women with urinary incontinence and risk factors for stress urinary incontinence in China. Menopause 2009;16:831-6.

2. Nygaard I, Barber MD, Burgio KL, et al. Prevalence of Symptomatic Pelvic Floor. JAMA 2008;300:1311-6.

3. Zhang L, Zhu L, Xu T, et al. A Population-based Survey of the Prevalence, Potential Risk Factors, and Symptomspecific Bother of Lower Urinary Tract Symptoms in Adult Chinese Women. Eur Urol 2015;68:97-112.

4. Espuña-Pons M, Fillol M, Pascual MA, et al. Female Pelvic Floor Dysfunction Research Group Pelvic floor symptoms and severity of pelvic organ prolapse in women seeking care for pelvic floor problems. Eur J Obstet Gynecol Reprod Biol 2014;177:141-5.

5. Grape HH, Dedering A, Jonasson AF. Retest reliability of surface electromyography on the pelvic floor muscles. Neurourol Urodyn 2009;28:395-9.

6. Yang ML, Wang Q, Yu XJ, et al. Pelvic floor function of 5143 women in early postpartum stage and analysis on the effect factors. Zhonghua Fu Chan Ke Za Zhi 2019;54:522-6.

7. Lukacz ES, Santiago-Lastra Y, Albo ME, et al. Urinary Incontinence in Women. JAMA 2017;318:1592.

8. Castro RA, Arruda RM, Bortolini MA. Female urinary incontinence: effective treatment strategies. Climacteric 2015;18:135-41.

9. Poli de Araújo M, Brito LGO, Rossi F, et al. Prevalence of Female Urinary Incontinence in Crossfit Practitioners and Associated Factors: An Internet Population-Based Survey. Female Pelvic Med Reconstr Surg 2020;26:97-100.

10. Stones RW, Padmadas SS, Guo S, et al. Dyspareunia, Urinary Sensory Symptoms, and Incontinence Among Young Chinese Women. Arch Sex Behav 2006;35:561-7.

11. Bardino M, Di Martino M, Ricci E, et al. Frequency and Determinants of Urinary Incontinence in Adolescent and Young Nulliparous Women. J Pediatr Adolesc Gynecol 2015;28:462-70.

12. Abitteboul Y, Leonard F, Mouly L, et al. Urinary incontinence in non-professional female marathon runners. Prog Urol 2015;25:636-41.

13. Brown SJ, Donath S, MacArthur C, et al. Urinary incontinence in nulliparous women before and during pregnancy: prevalence, incidence, and associated risk factors. Int Urogynecol J 2010;21:193-202.

14. Eliasson K, Nordlander I, Mattsson E, et al. Prevalence of urinary leakage in nulliparous women with respect to physical activity and micturition habits. Int Urogynecol J Pelvic Floor Dysfunct 2004;15:149-53.

15. Hagovska M, Śvihra J, Buková A, et al. Prevalence and risk of sport types to stress urinary incontinence in sportswomen: A cross-sectional study. Neurourol Urodyn 2018;37:1957-64.

16. Minassian VA, Drutz HP, Al-Badr A. Urinary incontinence as a worldwide problem. Int J Gynaecol Obstet 
2003;82:327-38.

17. Fiore HJSPE. Urinary Incontinence in Women: Evaluation and Management. Am Fam Physician 2019;100:339-48.

18. Shishido K, Peng Q, Jones R, et al. Influence of Pelvic

Cite this article as: Chang X, Ge H, Ye G, Quan X, Shen W, Zhang C, Huan M, Wu J. Analysis of pelvic floor electrical physiological parameters in nulliparous women with stress urinary incontinence. Transl Androl Urol 2021;10(4):1620-1626. doi: 10.21037/tau-20-1235
Floor Muscle Contraction on the Profile of Vaginal Closure Pressure in Continent and Stress Urinary Incontinent Women. J Urol 2008;179:1917-22. 